\title{
The AGN Properties of the Starburst Galaxy NGC 7582
}

\author{
T. V. Ricci ${ }^{1}$, J. E. Steiner ${ }^{1}$, R. B. Menezes ${ }^{1}$, A. Garcia-Rissmann ${ }^{2}$, \\ and R. Cid-Fernandes ${ }^{3}$ \\ ${ }^{1}$ Instituto de Astronomia, Geofísica e Ciências Atmosféricas, Universidade de São Paulo, \\ Rua do Matão, 1226, São Paulo SP, Brazil \\ Email: tiago@astro.iag.usp.br \\ ${ }^{2}$ Gemini Observatory \\ ${ }^{3}$ Universidade Federal de Santa Catarina
}

Keywords. galaxies: active, galaxies: starburst, galaxies: individual (NGC 7582)

NGC 7582 was identified as a starburst galaxy in the optical (Veron et al. 1981) but its X-ray emission is typical of a Seyfert 1 galaxy (Ward et al. 1978). We analyzed a datacube on this object obtained with the GMOS-IFU on the Gemini-South telescope. After a subtraction of the stellar component using the STARLIGHT code (Cid Fernandes et al. 2005), we looked for optical signatures of the AGN. We detected a broad $H \alpha$ component (Figure 1) in the source where Bianchi et al. (2007) identified the AGN in an HST optical image. We also found a broad $H \beta$ feature (Figure 2), but its emission reveals a extended source. We suggest that it is the light of the AGN scattered in the ionization cone. We propose that NGC 7582 is a Seyfert 1 galaxy. A number of other "hot-spots" and Wolf-Rayet features were also identified.

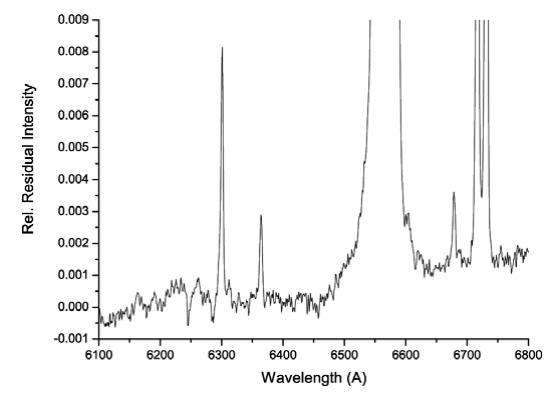

Figure 1. Spectra extracted from the region of the AGN.

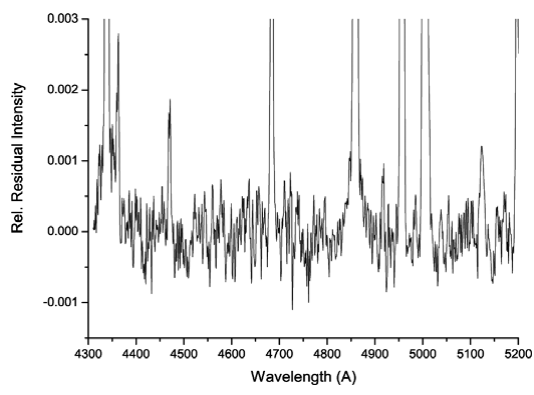

Figure 2. Spectra extracted from the region of the ionization cone.

\section{References}

Bianchi, S., Chiaberge, M., Piconcelli, E., \& Guainazzi, M. 2007, MNRAS, 374, 697

Cid Fernandes, R., Mateus, A., Sodré, L., Stasińska, G., \& Gomes, J. M. 2005, MNRAS, 358, 363

Veron, P., Veron, M. P., Bergeron, J., \& Zuidervijk, E. J. 1981, A\&A, 97, 71

Ward, M. J., Wilson, A. S., Penston, M. V., Elvis, M., Maccacaro, T., \& Tritton, K. P. 1978, ApJ, 223, 788 